\title{
Mind the Gap: Acetazolamide Prolonged Periods without Paralysis in a Girl with Andersen-Tawil Syndrome
}

\author{
Nina Žakelja Damjan Osredkar ${ }^{a, b}$ Nataša Šuštar ${ }^{a}$ \\ aDepartment of Pediatric Neurology, University Children's Hospital, University Medical \\ Centre Ljubljana, Ljubljana, Slovenia; ${ }^{b}$ Faculty of Medicine, Centre for Developmental \\ Neuroscience, University of Ljubljana, Ljubljana, Slovenia
}

\section{Keywords}

Andersen-Tawil syndrome $\cdot$ Ventricular arrhythmia $\cdot$ Muscle paralysis $\cdot$ Acetazolamide

\begin{abstract}
We present a case report of a 13-year-old girl with Andersen-Tawil Syndrome (ATS), a rare genetic disorder which is characterized by dysmorphic features, ventricular arrhythmias, and frequent episodes of muscle paralysis that interfere with daily activities and social engagement. After the introduction of off-label treatment with acetazolamide periods without paralysis lengthened, our patient became more independent of the help of her parents and required a wheelchair less frequently, thus improving her social life. Based on our experience, we recommend a trial of acetazolamide in patients with ATS.
\end{abstract}

\section{Introduction}

Andersen-Tawil Syndrome (ATS) is a rare potassium channelopathy, with an estimated prevalence of 1 per million [1]. It is characterized by a triad of (1) periodic paralysis and muscle weakness, (2) ventricular arrhythmias with a prolonged QT interval, and (3) dysmorphic features including low-set ears, broad forehead, small mandible, short stature, hypertelorism, clinodactyly or syndactyly, and scoliosis [2-4]. Periodic paralysis begins early in life, with individual episodes lasting from hours to days [1]. Several triggers have been associated with ATS, such as exercise or long periods of rest, cold, heat, emotional stress, and menstruation. However, paralysis can occur without obvious triggers $[2,5,6]$. Muscle strength usually returns to normal between episodes, although mild muscle weakness may eventually become permanent [5]. 
Approximately $60 \%$ of cases of ATS are caused by mutations in the KCNJ2 gene, while the cause of the majority of the remaining cases is still unknown [3]. In at least $50 \%$ of individuals ATS is hereditary, while the other half of patients have a de novo identified variant [2]. In most cases, ATS is caused by a heterozygous mutation of the KCNJ2 gene, which encodes an inward rectifier K+ channel (Kir2.1), expressed in the myocardium, skeletal muscle, brain, and osteocytes. This gene plays a key role in stabilizing the resting potential of the skeletal and cardiac muscles, which is critical for maintaining normal muscle function [3].

Because of the rarity of the syndrome, no randomized, controlled studies of therapeutic approaches have been conducted. Carbonic anhydrase inhibitors (acetazolamide and dichlorphenamide) have been used for almost 50 years as empiric treatment for hypo- and hyperkalemic episodes of periodic muscle paralysis, although their mechanism of action is still not fully understood. They act by promoting urinary excretion of potassium and bicarbonates, resulting in non-anion gap acidosis. Systemic acidosis has been proposed to reduce susceptibility to periodic paralysis [4]. Another potentially beneficial effect of acetazolamide may be that it promotes the opening of calcium-activated potassium channels which are found in skeletal muscle cells and could thus act as a surrogate for the Kir2.1 channel [5]. This could also be effective in treating permanent weakness by reducing intracellular sodium accumulation and thereby lessening muscle fiber damage. Approximately $50 \%$ of patients with ATS respond to this type of treatment [4, 7]. A retrospective study of the efficacy of acetazolamide in hypokalemic periodic paralysis concluded that genotype is an important factor in treatment response; however, no similar studies have been conducted in patients with ATS [7].

\section{Case Report/Case Presentation}

We present a 13-year-old girl with a genetically confirmed diagnosis of ATS. The girl was born in the 39th week of gestation of her mother's first pregnancy. Due to an irregular pulse on cardiotocography, labor was induced, and a caesarean section was performed. Her Apgar scores were 10/10/10. Her birth weight, length, and head circumference were at the 3rd, 10 th, and 5 th percentiles for gestational age, respectively. Immediately after birth, continuous electrocardiogram (ECG) monitoring revealed monomorphic ventricular extrasystoles, while echocardiogram showed a structurally normal heart with mild right ventricular dilatation. Due to intrauterine growth restriction, metabolic screening and abdominal and head ultrasounds were performed but revealed no abnormalities. Her neurological examination was unremarkable.

\section{Cardiac Symptoms}

Regular follow-up examinations were performed in the first years of her life. A 24-h Holter ECG monitoring revealed monomorphic ventricular extrasystoles, which required no treatment. At the age of 4, unstable polymorphic and biphasic ventricular tachycardia (VT) and prolonged QTc (up to $560 \mathrm{~ms}$ ) were discovered. She began treatment with propranolol which was gradually increased to the highest dose for her weight. As Holter monitoring continued to show polymorphic VT in the following months, magnesium citrate was introduced in addition to propranolol. In the following years, she was clinically stable, although frequent VTs remained present. At the age of 7 , flecainide was added to her existing therapy. This resulted in the normalization of ECG, which has remained normal to this day.

\section{Neurological Symptoms}

She first presented with neurological symptoms at the age of 2 years, showing signs of paroxysmal torticollis. On examination, dysmorphic features were noted, including low-set ears,

\section{Karger ${ }^{\prime}=$}


retrognathia, micrognathia, broad forehead, and clinodactyly. At the age of 5 years, she began to experience episodes of muscle cramps, pain, and weakness during which she could not walk up stairs normally. These episodes became more frequent when she started school. She reported difficulty walking after waking up, cramping, and pain in the muscles of her neck, back, and calves, and a compelling need to bend her head forward. The pain subsided when she rested and worsened when she resumed exercise after rest. The worst episodes usually lasted 3 days and occurred several times a year. The problems with walking typically worsened during the day, so that she was unable to walk or elevate her legs in the afternoon. She required assistance with personal hygiene and dressing. Neurological examinations performed during episodes of paralysis revealed proximal muscle weakness, most pronounced in the flexors and rotators of the trunk (3/5), gluteal muscles (2/5), and adductors of the lower limbs (3/5), a positive Trendelenburg sign, an awkward gait with inversion of the feet when she attempted to walk on her toes, and an inability to walk on heels. She could not actively abduct her arms and had to hold objects with both hands. Between these episodes of aggravation, her neurologic status was normal.

During the last 5 years, the frequency and severity of periodic muscle paralysis worsened. By the age of ten, the girl experienced episodes of muscle weakness every day, sometimes up to 5 times a day, especially at school after sitting for longer periods of time. Our patient never noticed any other triggers apart from long periods of resting our vigorous exercise and emotional stress. Her legs were affected more often than her arms, and she needed assistance to achieve a standing position and had to be carried up stairs. During the worst episodes, the muscle weakness affected her entire body, which included loss of control of her head and arms. She became increasingly dependent on her wheelchair and could no longer walk long distances or play with her peers.

\section{Diagnosis and Treatment}

The diagnostic workup included a variety of tests: blood tests performed during episodes of paralysis and in between them always showed normal electrolyte levels, including potassium; thyroid hormones, metabolic tests, and creatine kinase levels were normal on several occasions. Pompe disease was excluded by determining normal alpha-glucosidase activity in the blood spot. Electromyography was attempted multiple times, but the girl's fear of needles made the examination impossible. Somatosensory evoked potentials showed lower amplitudes of the popliteal neurogram and cortical somatosensory evoked potential after left tibial nerve stimulation. Magnetic resonance imaging of the brain showed some hyperintense changes in the periventricular white matter and no abnormalities in the medulla spinalis. Genetic analysis of the 16 genes related to long QT syndrome (AKAP9, ANK2, CACNA1C, CALM1, CALM2, CAV3, KCNE1, KCNE2, KCNH2, KCNJ2, KCNJ5, KCNQ1, NOSTAP, SCN4B, SCN5A, and SNTA1) revealed a mutation in the KCNJ2 gene, consistent with a diagnosis of ATS. Her parents were also tested for mutations in the KCNJ2 gene, but the tests were negative.

Acetazolamide is known to help patients with hypo- and hyperkalemic episodes of periodic muscle paralysis, but potassium imbalance was never documented in our patient, not during episodes of paralysis nor in between them. As her clinical picture worsened in the last few years, treatment with acetazolamide was started under clinical supervision, despite conflicting evidence of its efficacy in patients with ATS and reports that it may exacerbate the patients' neurological status [8]. One month after the introduction of $750 \mathrm{mg}$ of acetazolamide per day, without additional potassium supplements, the girl and her parents reported a significant reduction in the frequency and severity of muscle paralysis episodes. Although the muscle weakness still occurred daily, usually in the afternoon, it was more manageable and did not require assistance or wheelchair use. In the last 9 months of treatment with acetazolamide,

\section{Karger'}


she only had 2 severe episodes of paralysis that required the use of a wheelchair. She has also improved socially, as she can now play outside with her friends, cook, and is almost independent in personal care. Because her ECG has remained normal since the addition of flecainide to her treatment at the age of 7 years, we have not performed any further Holter monitoring since starting treatment with acetazolamide.

\section{Discussion}

We present the case of a girl with ATS that is unique in the fact that her cardiac problems preceded her neurological problems, as opposed to typical ATS, where episodes of muscle weakness usually occur at least 2 years prior to cardiac symptoms [5, 9]. In our patient, cardiac symptoms occurred immediately after birth and preceded episodes of paralysis by 2 years. Furthermore, in the course of her disease our patient experienced episodes of muscle paralysis on a daily basis, which is significantly more frequent than previously described, with a mean frequency of 3 episodes per month [7]. During these episodes, muscle weakness affected both her upper and lower extremities and was predominantly proximal, which is consistent with previous reports [5]. Episodes of muscle weakness can be associated with high (15\%), low (65\%), or normal (20\%) serum potassium levels; however, they have been found to be consistent within an individual patient $[7,9]$. In our patient, serum potassium levels were normal on every examination.

The diagnosis can be made if the patient has either 2 or more features of the triad or 1 feature and a family history of ATS [3]. Patients require a careful examination by a multidisciplinary team, including a cardiologist, neurologist, and geneticist. The baseline evaluation should include measurement of serum potassium and TSH levels, 12-lead ECG, 24-h Holter monitoring, and exercise stress test [2]. In our patient, laboratory tests were always normal, while 24-h Holter ECG monitoring revealed polymorphic and biphasic VT with prolonged QTc, in agreement with previous reports [5]. Genetic testing revealed a KCNJ2 gene mutation, which is the most common cause of ATS [3].

Treatment of episodic weakness in patients with hypokalemia or a relative drop in potassium levels is aimed at maintaining serum potassium levels at the upper end of the normal range by administration of oral potassium [2]. Elevated potassium levels are beneficial for cardiac symptoms as well, as they shorten the QTc interval and reduce cardiac arrhythmogenicity [1]. Light exercise may shorten or reduce the severity of the attack, which was confirmed in our patient's reports [2]. Empirical treatment with flecainide has been suggested in patients with severe, frequent ventricular arrhythmias [1,2]. Our patient's VT became fully suppressed after the introduction of flecainide into her treatment.

Although it is not a standard therapy for children with ATS, and despite reports of adverse effects in patients with ATS [8], acetazolamide was introduced into our patient's treatment, significantly improving her neurological status and providing her freedom of movement and social interactions that were previously severely hindered by her disease. Our patient did not experience any side effects of acetazolamide, the most common ones being paresthesia, fatigue, cognitive disturbances, and nephrolithiasis, which is a potential complication of treatment and should be monitored [7]. Antiarrhythmic drugs, particularly class I drugs, can paradoxically exacerbate the neuromuscular symptoms while prolonging the QT interval and should be avoided along with a myriad of other drugs known to do the same (e.g., tricyclic antidepressants, erythromycin, haloperidol, and others) [1,2]. Salbutamol inhalers and potassium-wasting diuretics should also be avoided, as the former may exacerbate cardiac arrhythmias and the latter may provoke QT interval prolongation [2]. However, based on our experience, we recommend a supervised trial of acetazolamide in patients with genetically confirmed ATS.

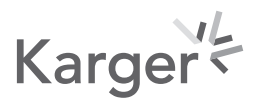




\section{Conclusion}

Multidisciplinary management is important in patients with ATS. Successful cardiac treatment allows survival, while treatment of periodic muscle paralysis contributes to improved daily function and greater independence in these patients. Improving the latter has a significant impact on the quality of life of patients with ATS. Based on our experience, we recommend a supervised trial of acetazolamide in patients with genetically confirmed ATS.

\section{Statement of Ethics}

The patient and her parents provided both oral and written informed consent for the publishing of this report. The paper is exempt from Ethical Committee approval as per guidelines by our National Ethics Committee. This is a case report study and does not include experimenting on animal and human subjects.

\section{Conflict of Interest Statement}

The authors have no conflicts of interest to declare.

\section{Funding Sources}

The authors did not receive any external funding.

\section{Author Contributions}

Nina Žakelj wrote the manuscript. Nataša Šuštar and Damjan Osredkar reviewed, edited, and approved the final version of the manuscript. Nataša Šuštar was the patient's primary neurologist.

\section{Data Availability Statement}

All data generated or analyzed during this study are included in this article. Further enquiries can be directed to the corresponding author.

\section{References}

1 Sansone V, Tawil R. Management and treatment of Andersen-Tawil syndrome (ATS). Neurotherapeutics. 2007 Apr;4(2):233-7.

2 Veerapandiyan A, Statland JM, Tawil R. Andersen-Tawil syndrome. GeneReviews ${ }^{\circledR}$. Seattle, WA: University of Washington; 1993.

3 Kokubun N, Aoki R, Nagashima T, Komagamine T, Kuroda Y, Horie M, et al. Clinical and neurophysiological variability in Andersen-Tawil syndrome. Muscle Nerve. 2019 Dec;60(6):752-7.

4 Statland JM, Fontaine B, Hanna MG, Johnson NE, Kissel JT, Sansone VA, et al. Review of the diagnosis and treatment of periodic paralysis. Muscle Nerve. 2018 Apr;57:522-30.

5 Nguyen HL, Pieper GH, Wilders R. Andersen-Tawil syndrome: Clinical and molecular aspects. Int J Cardiol. 2013;170(1):1-16.

6 Almuqbil M, Srour M. Child neurology: Andersen-Tawil syndrome. Neurology. 2015 Mar;84(11):e78-80.

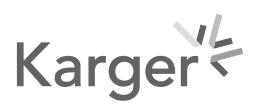


7 Matthews E, Portaro S, Ke Q, Sud R, Haworth A, Davis MB, et al. Acetazolamide efficacy in hypokalemic periodic paralysis and the predictive role of genotype. Neurology. 2011 Nov;77(22):1960-4.

8 Nagamine S, Sakoda S, Koide R, Kawata A, Yuan J, Takashima H, et al. A case of Andersen-Tawil syndrome presenting periodic paralysis exacerbated by acetazolamide. J Neurol Sci. 2014;347:385-6.

9 Smith AH, Fish FA, Kannankeril PJ. Andersen-Tawil syndrome. Indian Pacing Electrophysiol J. 2006 Jan;6(1): $32-43$. 